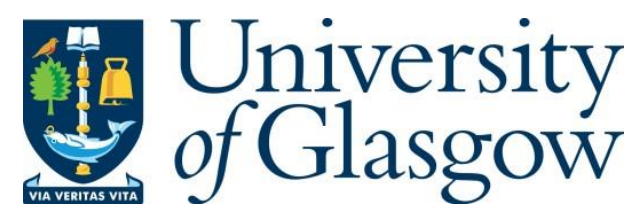

Kafi, M. A., Paul, A., Vilouras, A. and Dahiya, R. (2018) Chitosan-Graphene Oxide Based Ultra-Thin Conformable Sensing Patch for Cell-Health Monitoring. In: 2018 IEEE Sensors, New Delhi, India, 28-31 Oct 2018, ISBN 9781538647073.

There may be differences between this version and the published version. You are advised to consult the publisher's version if you wish to cite from it.

http://eprints.gla.ac.uk/196345/

Deposited on: 21 April 2020

Enlighten - Research publications by members of the University of Glasgow http://eprints.gla.ac.uk 


\title{
Chitosan-Graphene Oxide based ultra-thin conformable sensing patch for cell-health monitoring
}

\author{
Md. Abdul Kafi ${ }^{1,2}$, Ambarish Paul ${ }^{1}$, Anastasios Vilouras ${ }^{1}$, Ravinder Dahiya ${ }^{1 *}$ \\ ${ }^{1}$ Bendable Electronics and Sensing Technologies (BEST) Group, University of Glasgow, Glasgow G12 8QQ, UK, ${ }^{2}$ Department of \\ Microbiology and Hygiene, Bangladesh Agricultural University, Mymensingh, Bangladesh \\ *Correspondence to: Ravinder.Dahiya@glasgow.ac.uk
}

\begin{abstract}
The work presents a Chitosan-Graphene Oxide (Chi-GO) based ultra-thin sensors patch having arrays of gold (Au) based micro-gap $(60 \mu \mathrm{m})$ electrode. The cross-linked GO is shown to improve the stability of chitosan substrate in aqueous medium. With human dermal fibroblast (HDF) cell immobilized Chi-GO surface, the sensor patch has been evaluated for label free monitoring. The cyclic voltammetry (CV) of cell immobilized chiGO surface showed quasi-reversible nature with characteristic cathodic peak at $+300 \mathrm{mV}$ and anodic peak $-300 \mathrm{mV}$. Both peaks are stable and repeatable up to 50 -scan cycle without any potential shift. The device shows steady state peak enhancement (1.923$11.195 \mathrm{nA})$ with the growth period $(0-96 \mathrm{~h})$. The redox peak enhancement correlates with the proliferation rates of cell over time, indicating that it could be employed to investigate cytophysiologic state against any endo and exogenous stimulation. The new compatibility of cross-linked chitosan with microfabrication steps and the ability of presented sensor patch to monitor cellhealth will benefit a range of in vivo applications.
\end{abstract}

Keywords-Chitosan, Graphene Oxide, HDF Cell, Cyclic Voltammetry.

\section{INTRODUCTION}

Chitosan holds significant promise for several biomedical and tissure engineering applications owing to its attractive properties such as self-renewability, auto degradability, bioresorbability and biocompatibility [1]. It has already been utilized in wound dressing, as drug carrier, and scaffold for tissue engineering etc. [2]. Recently, this promising material has also been used in biosensors that measure wide ranging analytes from biological [3], chemical [4] and environmental sources [5]. The attractive properties of chitosan are promising for some application and bring challenges for other. For example, the due to degradability in aqueous medium the chitosan-based biosensors have been primarily used for in-vitro measurement, even if other properties make this material attractive for in-vivo application as well. If the degradability issues can be handled, for example by tuning the chitosan surface properties, then this promising material can open an unexplored area i.e. chitosanbased sensors for in vivo application. Among few methods that have been explored recently to overcome the issue of stability of chitosan, the use of glutaraldehyde and Graphene Oxide (GO) are noteworthy. For example, with glutaraldehyde-chitosan cross-linking it has been possible to develop stable scaffold for $3 \mathrm{D}$ growth and proliferations of cell in tissue engineering [2]. In

This work was supported by the Marie Curie Individual Fellowship grant (BEND,H2020-MSCA-IF-2015, 704807, Newton International fellowship, and EPSRC Engineering Fellowship for Growth - PRINTSKIN (EP/M002527/1) the case of $\mathrm{GO}$, the crosslinking between carboxyl $(-\mathrm{COOH})$ group of chitosan and hydroxyl $(-\mathrm{OH})$ group of $\mathrm{GO}$ has been shown to improve the stability [6]. The chitosan-GO (chi-GO) surface also offer suitable condition for metallization, which helps to realise electrodes to measure cellular redox.

The incompatibility of chitosan substrate with the microfabrication steps (e.g. metallization difficulties) has been a major challenge with the state-of-the-art chitosan-based platforms. Considering this issue, the possibility of metallization of Chi-GO offers an interesting opportunity to develop biosensors to understand and correlate the endogenous and exogenous influences, develop better understanding of cellhealth and hence improve diagnostic practise $[7,8]$. Due to body organs being soft and curvy, the in-vivo applications also require the Chi-GO sensors to be flexible and conformable. In the regard, this work takes advantage of recent advances in flexible and stretchable electronics technology [9-12] to develop ChiGO based ultra-thin and highly conformable sensors patch. The developed patch comprises of an array of biosensors made from gold $(\mathrm{Au})$ based electrodes with micro-gap $(60 \mu \mathrm{m})$ bridged with cells. The viability of using the sensors patch for label free
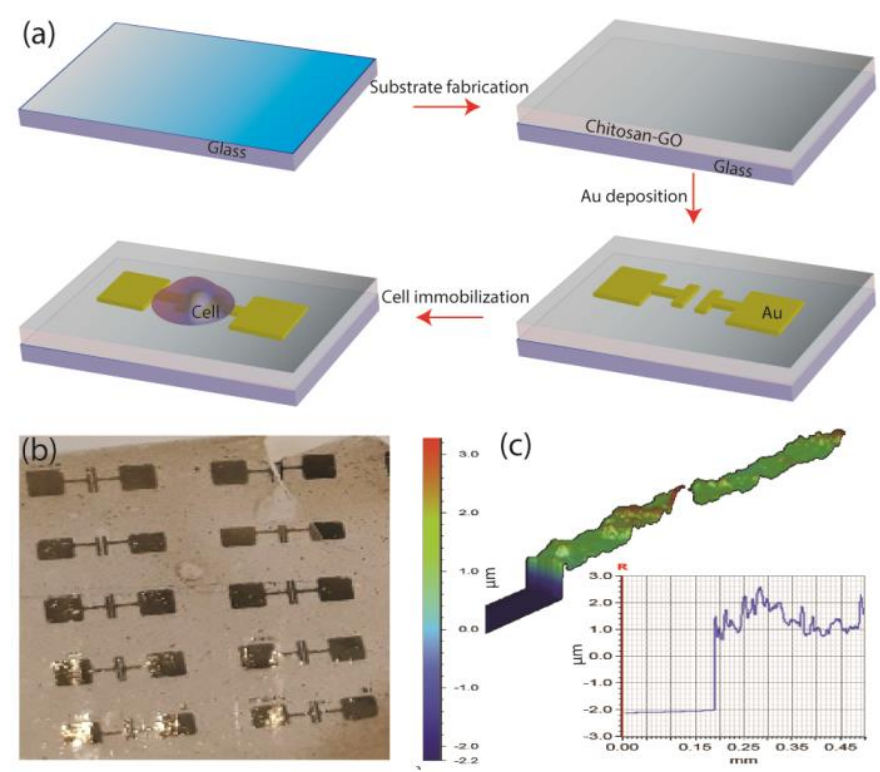

Figure 1. (a) Schematics of Chitosan-GO substrate fabrication, (b) as fabricated micro-gap $(60 \mu \mathrm{m})$ on chitosan-GO and (c) Dektak XT profilometer map of Chitosan-GO film on planar substrate where line profile showing the thickness of the film. 

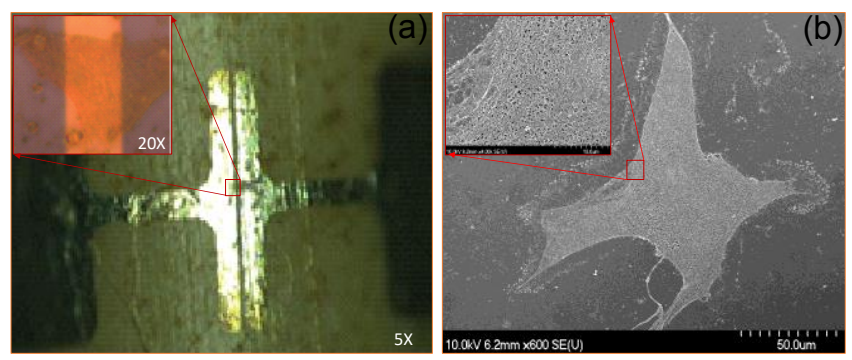

Figure 2. Cell immobilized micro-gap: (a) Optical image with magnified image obtained from paraformaldehyde fixed dehydrated sample in the inset; (b) SEM image (inset shows the magnified image)

monitoring of human dermal fibroblast (HDF) has been demonstrated.

This paper is organised as follows: The detailed related to fabrication and characterization of Chi-GO based biosensor are given in Section II. This is followed by analysis of results from experiments in Section III. Finally, the key results are summarized in Section IV.

\section{FABRICATION AND CHARACTERIZATION}

\section{A. Materials and equipments}

Chitosan powder obtained from Sigma-Aldrich was added in continuously stirred DIW to achieve homogenous suspension. The jelly like chitosan solution was achieved by adding acetic acid dropwise while stirring. Crosslinking was performed by adding GO (Graphene Laboratories Inc. NY, USA) in the chitosan solution at a ratio of 1:16 during stirring. The homogenous Chi-GO suspension was achieved after overnight stirring. The ultrathin Chi-GO substrate was achieved by casting it on a clean glass slide. A thin smooth chi-GO substrate was achieved after curing over light at RT. A thin layer of gold (Au) was evaporated on the Chi-GO substrate using e-beam and hard mask to achieve micro-gap electrode.

\section{B. Morpholigical characterization}

The fabricated Chi-GO substrate was realized with Scanning Electron Microscopy (SEM) and Dektak XT profilometer. For SEM investigation the substrate was placed on silicon wafer and

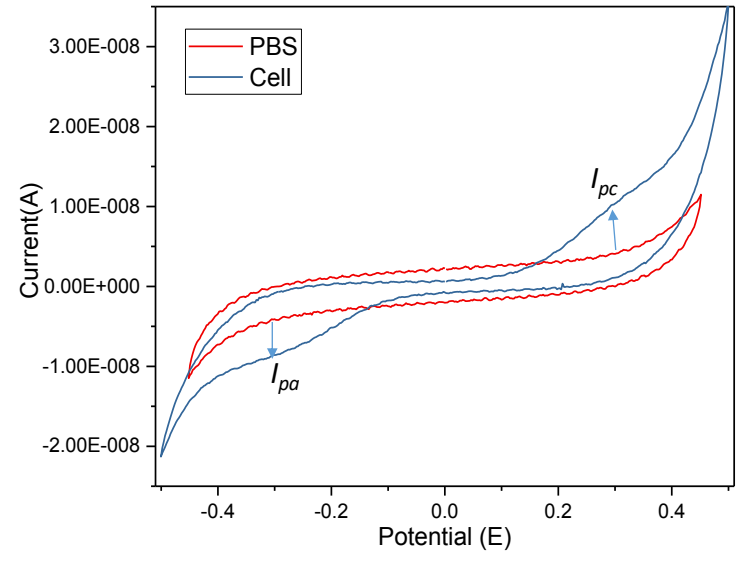

Figure 3. Redox behavior of HDF cell immobilized Chi-GO substrate. CV measured using PBS $(0.01 \mathrm{M}, \mathrm{pH} 7.4)$ as electrolyte at a scan rate of $50 \mathrm{mVs}^{-1}$. All the experiment was conducted at RT. The experiment was repeated three times with maintaining identical condition. a thin layer of gold $(\mathrm{Au})$ was sputtered on it prior to imaging. Images were obtained with field emission scanning electron microscope (Hitachi S-4700) at an accelerated voltage of $10 \mathrm{KV}$ and $10 \mathrm{~mA}$ current, whereas profilometric analysis was performed using Bruker Dektak XT height profiler. For optical imaging the Chi-GO was placed on glass slide and images were obtained from Nikon Eclipse LV100ND microscope connected with Leica MC170HD camera.

\section{Cell culture and maintinance}

Human dermal fibroblast (HDF) cells were purchased from GIBCOC and seeded at density of $5 \times 10^{3} \mathrm{cell} / \mathrm{cm}^{2}$ in DMEM supplemented with $10 \%$ foetal bovine serum and $1 \%$ antibiotic and antimycotic and kept at $37^{\circ} \mathrm{C}$ in a standard cell culture incubator. The cell was fed twice in a week and subculture performed when $100 \%$ confluency attain. Cell from 3rd passage was employed for all experiments in this research.

\section{Electrophysiological investigation}

For electrophysiological investigation, the Au micro-gap patterned electrodes on chi-GO substrate was seeded with HDF cell at a concentration of $1.5 \times 10^{4}$ cell per device and kept at $37^{\circ} \mathrm{C}$ in a humidified atmosphere with $5 \% \mathrm{CO}_{2}$ for $48 \mathrm{~h}$. Then the device was washed with phosphate buffer saline (PBS , $\mathrm{pH}$ 7.4) and $\mathrm{CV}$ investigations were carried out with a standard potentiostat (Metrohm Autolab B.V., Kanaalweg 29-G 3526 KM Utrecht, Netherland). All experiments were performed in triplicate using freshly prepared device at an identical condition.

\section{E. Cell viability study}

Viability of HDF cells attached on the micro-gap electrode was determined by studying the redox peak intensities. To this end, the cells were seeded on the batches of device and allowed to grow for $0,24,48,72$ and $96 \mathrm{~h}$. Electrochemical investigation was performed on representative samples from each time point. All the measurements were repeated at least three times and the error bars have been shown in the figures.

\section{EXPERIEMENTAL RESULTS}

\section{A. Ultra-thin chi-GO cell chip}

The ultra-thin Chi-GO layer was fabricated on silicon wafer by drop casting method (Fig. 1a). The fabricated Chi-GO layer

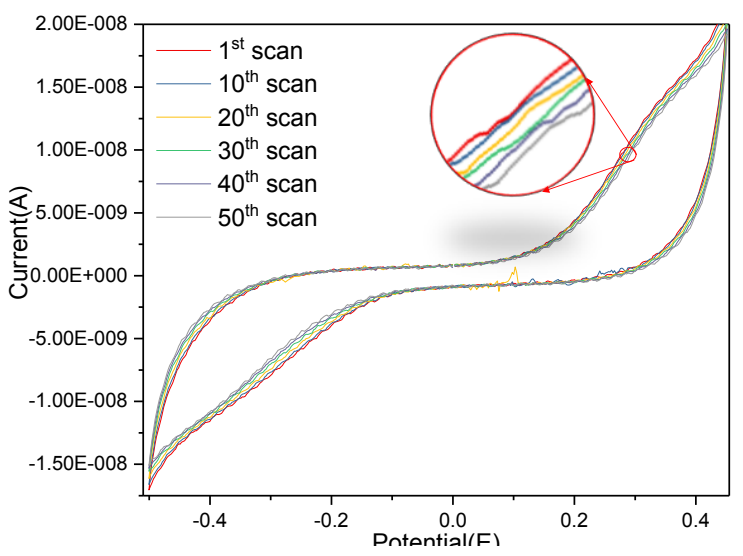

Figure 4. CV of HDF immobilized Chi-GO substrate at various scan cycle. CV was measured using PBS (0.01 M, pH 7.4) as an electrolyte at a scan rate of 50 $\mathrm{mVs}^{-1}$. The experiment was repeated three times with identical condition. 
attains stability to ordinary solvent. This was achieved by crosslinking of chitosan monomer through interaction between its carboxyl $(-\mathrm{COOH})$ group and hydroxyl $(-\mathrm{OH})$ group of $\mathrm{GO}$ [6]. This cross-linked Chi-GO substrate allows successful metallization to realize Au micro-gap electrodes (Fig. 1b). The micro-gap electrode modified substrate showed enough stability to cell culture and their maintenance. The fabricated chitosan substrate was thin $(2.8 \mu \mathrm{m})$ enough to conform to any shape, allowing their use in in-vivo application (Fig. 1c). To prove this, the HDF cell was immobilized on the Chi-GO substrate and maintained for electrochemical investigation. The cell immobilization was confirmed with optical (Fig. 2a) as well as SEM imaging (Fig. 2b). The HDF cell firmly adhered to the ChiGO substrate through focal adhesion and overcome dissociation during the washing step prior to the electrochemical measurement [9]. The focal adhesion is evident from SEM images shown in Fig. 2b. The micro-gap electrode bridging, which is critical for voltammetric analysis in this study, was confirmed with the optical images shown in the inset of Fig. 2 a.

\section{B. Electrochemical characterization of $H D F$}

The cell immobilized substrate was subjected to electrochemical investigation using $\mathrm{CV}$ at scan rate of $50 \mathrm{mV} / \mathrm{s}$ with a potential window of $+500 \mathrm{mV}$ to $-500 \mathrm{mV}$. The cellimmobilized platform was washed thrice with PBS and measurements were performed in PBS (PBS, pH 7.4) at RT. The $\mathrm{CV}$ obtained from cell cultured Chi-GO substrate showed a quasi-reversible redox peak with cathodic peak $\left(I_{p c}\right)$ at $+300 \mathrm{mV}$ and anodic peak $\left(I_{p a}\right)$ at $-300 \mathrm{mV}$ (Fig. 3). The absence of such peak from a device without cell confirms that redox is originated from the immobilized HDF. Considering quasi-reversibility, the peak potential difference between $I_{p c}-I_{p a}$ is $\geq 100 \mathrm{mV}$ and the current ratio $I_{p c} / I_{p a}$ is $\geq 1[13,14]$. The redox peak showed stability to scan rates and scan cycle. There was no significant difference in the current peak up to 50-scan cycle (Fig. 4). This indicates that the electrical signal are stable and repeatable. Considering stability and repeatability redox peak, together with mechanical properties discussed earlier, the device is suitable for in vivo application.

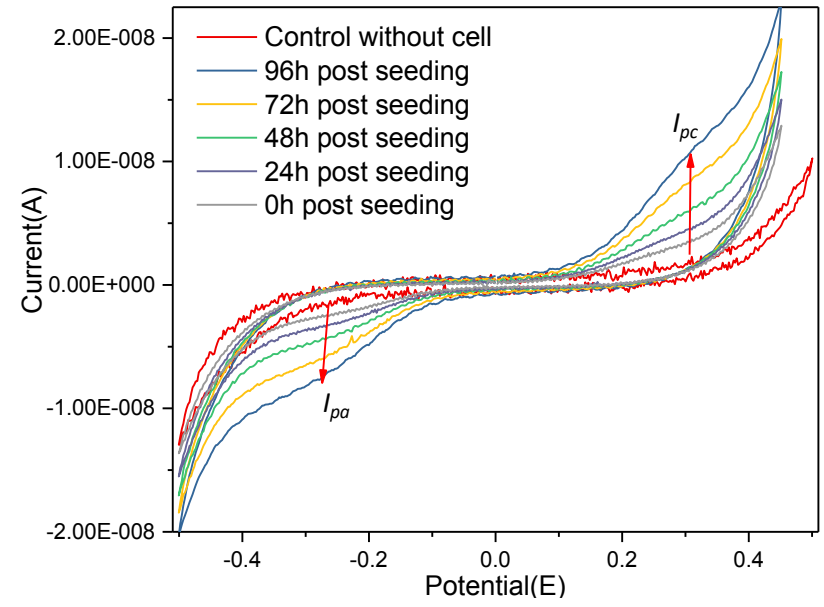

Figure 5. CV of HDF immobilized Chi-GO substrate at various peroid after seeding. $\mathrm{CV}$ was measured using PBS $(0.01 \mathrm{M}, \mathrm{pH} 7.4)$ as an electrolyte at a scan rate of $50 \mathrm{mVs}^{-1}$. The experiment was repeated three times with maintaining identical condition.

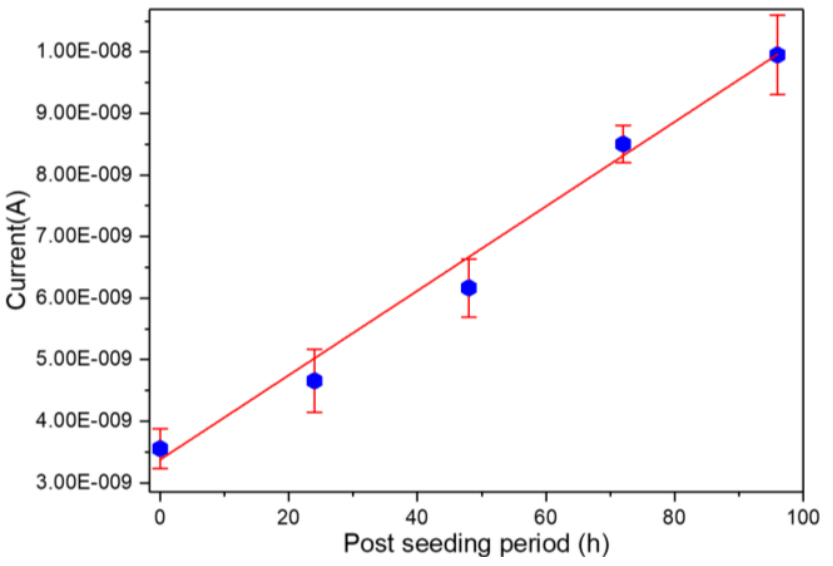

Figure 6. Changes in redox peak intensities corresponding to periods of cell growth on the Chi-GO substrate. The linear increases in current peaks $\left(I_{\mathrm{pc}}\right)$ in a concentration-dependent manner $\left(\mathrm{R}^{2}=0.989\right)$. Data are the mean \pm standard deviation of three different experiments.

\section{Cell health monitoring}

The device was employed for voltammetric monitoring of cell proliferation over the growth period of $96 \mathrm{~h}$. The CV peak intensities became prominent with increased cell growth period (Fig. 5). Both $I_{p c}$ and $I_{p a}$ peak showed similar trend of enhancement without any peak shift. Hence, both peak values obtained from voltammogram could be considered as sensing values. Herein, a concentration dependent linear plot $\left(\mathrm{R}^{2}=\right.$ 0.981 ) was obtained between the current intensities $I_{p c}$ and DA concentrations (Fig. 6). The peak enhancement is due to the redox of cell that bridges the micro-gape electrode. It is well known that living cell has distinct cell line and cell cycle stage specific redox property $[8,15]$. Here, the peak is derived from the heathy cell that remained attached with electrode since dead are not able to bridge the gap [16]. Hence, the measured redox reflects the viable cell population only. The changes in peak intensity reflect the state of cell health to any exogenous or endogenous influences affecting the cell viability. Therefore, the presented device is able to monitor cyto-physiologic state by analyzing and quantifying the redox signal.

\section{CONCLUSION}

A Chi-GO based ultra-thin bio-platform is presented here for cell-health monitoring using a label free electrochemical method. To this end, an array of micro-gap electrodes was fabricated by depositing gold ( $\mathrm{Au}$ ) on Chi-GO substrate and Human dermal fibroblast (HDF) cell was immobilized on the micro-gap. The CV of the cell-immobilized sensor shows a quasi-reversible redox with characteristic cathodic peak $\left(I_{p c}\right)$ and anodic peak $\left(I_{p a}\right)$ at $+300 \mathrm{mV}$, and $-300 \mathrm{mV}$, respectively. The intensities of both redox peaks were enhanced (1.923$11.195 \mathrm{nA}$ ) with the growth period of HDF cell without potential shift. The changes in peak intensities reflect the numbers of healthy cell attached on the electrode (0-96h). A linear plot $\left(\mathrm{R}^{2}=0.981\right)$ derived from $I_{p c}$ vales with post seeding period shows that the device is capable of monitoring cell health by analysing and quantifying the redox peak intensities. The conformability and tuneable degradability of presented bioplatform is likely to advance many biomedical applications. 


\section{REFERENCES}

[1] W. Suginta, P. Khunkaewla, A. Schulte, "Electrochemical Biosensor Applications of Polysaccharides Chitin and Chitosan" Chem. Rev. Vol. 113, pp. 5458-5479, 2013.

[2] M. A. Kafi, P. Yos, Y. Nakamuta, M. Todo, "Proliferation Behavior of Mesenchymal Stem Cells in Peptide Functionalized Chitosan Scaffolds," IFMBE proceedings vol. 43, pp. 279-282, 2014.

[3] M. A. Kafi, A. Paul, R. Dahiya, "Graphene oxide-chitosan based flexible biosensor" IEEE SENSORS-2017. DOI:10.1109/ICSENS.2017.8234441

[4] K.V. Ragavan, S. R. Ahmed, X. Weng, S. Neethirajan, "Chitosan as a peroxidase mimic: Paper based sensor for the detection of hydrogen peroxide" Sens. Actuators B Chem. Vol. 272 pp. 8-13, 2018.

[5] Y. W. Fen, W. M. M. Yunus, N. A. Yusof, "Surface plasmon resonance optical sensor for detection of $\mathrm{Pb} 2+$ based on immobilized p-tertbutylcalix[4]arene-tetrakis in chitosan thin film as an active layer" Sens. Actuators B Chem.Vol. 171-172, pp. 287-293, 2012.

[6] L. He, H. Wang, G. Xia, J. Sun, R. Song, "Chitosan/graphene oxide nanocomposite films with enhanced interfacial interaction and their electrochemical applications" Appl. Surf. Sci. Vol. 314, pp. 510-515, 2014.

[7] S. Neethirajan, "Recent advances in wearable sensors for animal health management" Sens. Biosensing Res. Vol.12, pp. 15-29, 2017.

[8] M. A. Kafi, T. -H. Kim, J. -W. Choi, "Cell Chip to Analyze Cell lines and Cell Cycle Stages Based on Electrochemical Method" SENSORDEVICES 2011: The Second International Conference on Sensor Device Technologies and Applications, pp. 147-150, 2011. ISBN: 978-1-61208-145-8.
[9] W. Dang, L. Manjakkal, W. T. Navaraj, L. Lorenzelli, V. Vinciguerra, R. Dahiya, "Stretchable wireless system for sweat $\mathrm{pH}$ monitoring. Biosens. Bioelectron. Vol 107, pp. 192-202, 2018.

[10] R. S. Dahiya, "Epidermial electronics: flexible electronics for biomedical applications. In: Carrara, S. and Iniewski, K. (eds.) Handbook of Bioelectronics: Directly Interfacing Electronics and Biological Systems. Cambridge University Press: Cambridge, pp. 245-255, 2015. ISBN 9781107040830

[11] S. Gupta, W. T. Navaraj, L. Lorenzelli, R. Dahiya, "Ultra-Thin Chips for High-Performance Flexible Electronics," NPJ Flexible Electronics, Vol 2, Art. 8, doi:10.1038/s41528-018-0021-5, 2018.

[12] W. Dang, V. Vinciguerra, L. Lorenzelli, R. Dahiya, "Printable Stretchable Interconnects," Flexible and Printed Electronics, Vol. 2, 013003, 2017.

[13] M. A. Kafi, T. -H. Kim, T. Lee, J.-W. Choi, "Cell Chip with Nano-Scale Peptide Layer to Detect Dopamine Secretion from Neuronal Cells," J. Nanoscie. Nanotech. vol. 11, pp. 1-5, 2011.

[14] M. H. Bridge, E. Williams, M. E. G. Lyons, K. F. Tipton, W. Linert, "Electrochemical investigation into the redox activity of $\mathrm{Fe}(\mathrm{II}) / \mathrm{Fe}(\mathrm{III})$ in the presence of nicotine and possible relations to neurodegenerative diseases," Biochim. Biophys. Acta. vol. 1690, pp. 77-84, 2004.

[15] M. A. Kafi, T. -H. Kim, J. H. An, J. -W. Choi, "Electrochemical cellbased chip for the detection of toxic effects of bisphenol-A on neuroblastoma cells" Biosens. Bioelectron. Vol. 26, pp. 3371-3375, 2011.

[16] N. Mochizuki, T. Kakegawa, T. Osaki, N. Sadr, N. N. Kachouie, H. Suzuki, J. Fukuda, "Tissue engineering based on electrochemical desorption of an RGD-containing oligopeptide" J. Tissue Eng. Regen. Med. Vol. 7, pp. 236-243, 2013. 